\title{
Lettre à la rédaction / Letter to the Editor
}

\section{L'hémodialyse est utile en cas d'intoxication grave au baclofène}

\author{
Haemodialysis is useful after baclofen intoxication \\ Erwan d'Aranda ${ }^{\star}$, Guillaume Lacroix, Jean Cotte, Pierre-Julien Cungi, Eric Meaudre \\ Service de réanimation, Hôpital d'Instruction des Armées Sainte-Anne, Boulevard Sainte Anne, BP 20545, 83041 Toulon Cedex 09, France
}

Mots clés : Baclofène, insuffisance rénale, intoxication, hémodialyse

Key words: Baclofen, intoxication, renal insufficiency, haemodialysis

Reçu le 28 novembre 2012, accepté après modifications le 30 mars 2013

Publication en ligne le 13 juin 2013

Le baclofène est un médicament utilisé dans le traitement de la spasticité musculaire. Son utilisation a été élargie depuis 2008 hors AMM dans le traitement des addictions, à l'éthanol principalement $[1,2]$. Les doses potentiellement utilisables sont nettement supérieures à celles utilisées dans le traitement de la spasticité. Le surdosage d'un traitement par baclofène peut engager le pronostic vital, et ce risque s'accroît avec l'élargissement de son utilisation. Nous rapportons un cas d'intoxication par le baclofène survenu chez un patient éthylique et insuffisant rénal.

\section{Observation}

Un patient de 58 ans, éthylo-tabagique chronique, avait pour antécédents : une broncho-pneumopathie chronique obstructive, une cardiopathie alcoolique, une insuffisance rénale chronique sévère post néphrectomie tumorale gauche et un syndrome dépressif. Il était traité par bisoprolol, atorvastatine, escitalopram, acamprosate et naltrexone. Le patient était adressé à l'hôpital par son médecin traitant pour trouble de la vigilance, quelques jours après le début d'une nouvelle cure de désintoxication à l'éthanol et l'introduction d'un traitement par baclofène à la posologie de $70 \mathrm{mg}$ par jour per os. Aux urgences de l'hôpital, le patient présentait un état d'agitation et un syndrome confusionnel fluctuant avec des troubles de la conscience (score de Glasgow à 10), sans signe de focalisation neurologique, quelques épisodes de fasciculations des membres inférieurs et une $\mathrm{SpO}_{2}$ à $95 \%$ en air. L'examen respiratoire retrouvait une fréquence respiratoire à $13 / \mathrm{min}$, des ronchis des bases et une acidose respiratoire $\left(\mathrm{pH}=7,17, \mathrm{PaCO}_{2} 66 \mathrm{mmHg}, \mathrm{HCO}_{3}^{-} 23 \mathrm{mmol} / \mathrm{L}\right.$,

\footnotetext{
^Correspondance : Erwan d'Aranda, erwanm3d@gmail.com
}

lactate $0,6 \mathrm{mmol} / \mathrm{L})$. Les constantes hémodynamiques étaient normales (pression artérielle à 139/78 $\mathrm{mmHg}$, fréquence cardiaque à $85 / \mathrm{min}$ ). Il était apyrétique à $36,7^{\circ} \mathrm{C}$. Le bilan biologique retrouvait : un ionogramme normal, une insuffisance rénale chronique sévère à diurèse préservée avec une clairance de Cockcroft à $28 \mathrm{~mL} / \mathrm{min}$, des enzymes hépatiques normales, une ammoniémie normale à $24 \mu \mathrm{mol} / \mathrm{L}$, une troponine $\mathrm{T}$ inférieure à $0,01 \mu \mathrm{g} / \mathrm{L}$, un NT pro BNP augmenté à $11900 \mathrm{ng} / \mathrm{L}$ et une alcoolémie nulle. L'ECG était sans anomalie. La radiographie pulmonaire ne montrait pas d'infiltrat alvéolo-interstitiel. Le bilan neurologique comprenait une tomodensitométrie cérébrale et une ponction lombaire qui étaient normales. L'électroencéphalogramme objectivait un ralentissement diffus. Devant ce tableau atypique de souffrance cérébrale et une acidose hypercapnique sans polypnée compensatrice faisant évoquer une absence de stimulation des centres respiratoires centraux, le centre antipoison de la région était contacté pour une probable encéphalopathie médicamenteuse. Il confirmait la concordance des symptômes avec une intoxication possible au baclofène et la possibilité d'un traitement par hémodialyse. Le patient était admis en réanimation. La décision d'une séance d'hémodialyse était prise devant l'absence d'argument pour une autre intoxication (alcoolémie nulle, pas de modification de son traitement de fond), une dose quotidienne de baclofène 14 fois supérieure à la dose recommandée par l'ANSM et une symptomatologie pouvant persister plus de $72 \mathrm{~h}$ chez les patients à fonction rénale normale en cas de surdosage. Le dosage sanguin du baclofène, disponible a posteriori, était de $631 \mu \mathrm{g} / \mathrm{L}$ (zone thérapeutique : 80 à $400 \mu \mathrm{g} / \mathrm{L}$ ). Une séance d'hémodialyse d'une durée de $3 \mathrm{~h}$ était entreprise avec une évolution clinique spectaculaire : les symptômes neurologiques disparaissaient, la dépression respiratoire s'amoindrissait avec une augmentation de la fréquence 
respiratoire et une correction de l'acidose sur la gazométrie. Le patient sortait de la réanimation à $\mathrm{H}+12$ et de l'hôpital à $\mathrm{H}+48$.

\section{Discussion}

Le baclofène est un agoniste des récepteurs GABA B du système nerveux central et périphérique. Il est utilisé de façon courante par voie orale et intrathécale dans le traitement de la spasticité musculaire d'origine centrale. Le baclofène est un médicament neurotrope, liposoluble permettant le passage de la barrière hémato-encéphalique. Son absorption est digestive. Il a une faible fixation protéique $(30 \%)$, un faible volume de distribution $0,7 \mathrm{~L} / \mathrm{kg}$, et une demi-vie courte de $6-8 \mathrm{~h}$. Il présente un faible métabolisme hépatique en un métabolite inactif et son élimination sous forme inchangée est rénale à $85 \%$, principalement par filtration glomérulaire $[3,4]$. Ces caractéristiques expliquent le risque d'accumulation en cas d'insuffisance rénale et le caractère dialysable en cas d'intoxication comme dans notre cas. La zone thérapeutique plasmatique est comprise entre 80 et $400 \mu \mathrm{g} / \mathrm{L}$, mais le dosage plasmatique du baclofène n'est pas réalisable en routine et en limite l'intérêt à une confirmation a posteriori de l'intoxication. Dans le traitement de la spasticité, la posologie optimale est habituellement de 40 à $80 \mathrm{mg}$ en 3 fois par jour. Au-delà, les neurologues peuvent recourir à la voie intrathécale qui a reçu son AMM pour la spasticité réfractaire au baclofène par voie orale. Chez l'insuffisant rénal, il est conseillé de commencer à seulement $5 \mathrm{mg}$ par jour. El-Husseini a analysé 42 patients intoxiqués au baclofène et insuffisants rénaux; il recommande de ne pas utiliser le baclofène chez les patients avec une clairance inférieure à $30 \mathrm{~mL} / \mathrm{min}$ [5]. Dans le traitement des addictions à l'éthanol, les posologies per os optimales sont parfois nettement supérieures avec une moyenne quotidienne de $145 \mathrm{mg}$ dans l'essai d'Ameisen [6]. Ainsi, les effets secondaires fréquents mais peu graves observés lors du traitement de la spasticité risquent d'augmenter en nombre et en gravité. Les effets secondaires les plus courants du baclofène par voie orale sont la somnolence et la faiblesse musculaire, réversibles en 24/48 h après diminution ou arrêt du médicament. En cas de surdosage, il existe principalement une dépression du système nerveux central avec une hypotonie, une hyporéflexie, des troubles de la conscience, jusqu' au coma avec perte des réflexes du tronc cérébral pouvant mimer un état de mort cérébrale [2,7]. Des myoclonies ou des crises comitiales peuvent être observées et le tracé électroencéphalographique peut montrer un aspect de «burst-suppression» ou des anomalies paroxystiques diffuses pseudopériodiques [8]. Une dépression respiratoire est fréquente et des anomalies cardiovasculaires peuvent être présentes. Malgré la présence de signes de gravité, il existe une faible morbi-mortalité liée au surdosage en baclofène. Chez les 107 patients de Weißhaar et coll. [9] et les 23 patients de Leung et coll. [10], intoxiqués à doses massives (jusqu'à 2,5 g), seuls 3 décès ( 1 par œdème cérébral et 2 non liés directement à l'intoxication) ont été rapportés, et il n'existait pas de séquelles à la sortie de l'hôpital. Les symptômes secondaires à l'intoxication sont rapidement réversibles après élimination du médicament. Il n'existe pas d'antidote. Le traitement est principalement symptomatique par assistance des défaillances d'organes. L'hémodialyse n'est pas systématiquement indiquée dans l'intoxication au baclofène mais est à discuter devant la gravité du tableau clinique et/ou en cas d'insuffisance rénale comme dans notre observation. L'hémodialyse permet de diminuer significativement la demi-vie du baclofène, de $15,5 \mathrm{~h}$ à $2,06 \mathrm{~h}$ pour $\mathrm{Wu}$ et coll. [3]. Notre cas montre qu'une courte séance d'hémodialyse permet une amélioration clinique rapide.

Le baclofène est un traitement en plein essor dont le surdosage peut engager le pronostic vital et ce, d'autant que les patients sont insuffisants rénaux.

\section{Conflits d'intérêts. Aucun.}

\section{Références}

1. Baclofène et traitement de l'alcoolo-dépendance : l'ANSM autorise deux essais cliniques. 2012. http://ansm.sante.fr.

2. Effets indésirables du baclofène dans le traitement des addictions Suivi national de pharmacovigilance. 2012. http://ansm.sante.fr.

3. Wu VC, Lin LS, Lin SM, Fang CC. Treatment of baclofen overdose by haemodialysis: a pharmacokinetic study. Nephrol Dial Transplant. 2005; 20: 441-443.

4. Brvar M, Vrtovec M, Kovac D, Kozelj G, Pezdir T, Bunc M. Haemodialysis clearance of baclofen. Eur J Clin Pharmacol. 2007; 63: 1143-1146.

5. El-Husseini A, Sabucedo A, Lamarche J, Courville C, Peguero A. Baclofen toxicity in patients with advanced nephropathy: proposal for new labeling. Am J Nephrol. 2011; 34: 491-495.

6. Ameisen O, de Beaurepaire R. Suppression de la dépendance à l'alcool et de la consommation d'alcool par le baclofène à haute dose : un essai en ouvert. Ann Med Psychol. 2010; 168: 159-162.

7. Sullivan R, Hodgman MJ, Kao L, Tormoehlen LM. Baclofen overdose mimicking brain death. Clin Toxicol (Phila). 2012; 50: $141-144$.

8. Rochart N, Berger P, Brochet-Paille A, Poiron L, Chillet P. Intoxication aiguë au baclofène : places de l'EEG et de la baclofènémie ? Journal Européen des Urgences et de Réanimation. 2012; 24: 54-59.

9. Weißhaar GF, Hoemberg M, Bender K, Bangen U, Herkenrath P, Eifinger F. Baclofen intoxication: a "fun drug" causing deep coma and nonconvulsive status epilepticus-a case report and review of the literature. Eur J Pediatr. 2012; 171(10): 1541-1547.

10. Leung NY, Whyte IM, Isbister GK. Baclofen overdose: defining the spectrum of toxicity. Emerg Med Australas. 2006; 18: $77-82$. 